Kragujevac Journal of Mathematics

Volume 45(5) (2021), Pages 751-759.

\title{
EIGENVALUES OF CIRCULANT MATRICES AND A CONJECTURE OF RYSER
}

\author{
REINHARDT EULER ${ }^{1}$, LUIS H. GALLARDO ${ }^{2}$, AND OLIVIER RAHAVANDRAINY ${ }^{2}$
}

\begin{abstract}
We prove that there is no circulant Hadamard matrix $H$ with first row $\left[h_{1}, \ldots, h_{n}\right]$ of order $n>4$, under some linear conditions on the $h_{i}$ 's. All these conditions hold in the known case $n=4$, so that our results can be thought as characterizations of properties that only hold when $n=4$. Our first conditions imply that some eigenvalue $\lambda$ of $H$ is a sum of $\sqrt{n}$ terms $h_{j} \omega^{j}$, where $\omega$ is a primitive $n$-th root of 1 . The same conclusion holds also if some complex arithmetic means associated to $\lambda$ are algebraic integers (second conditions). Moreover, our third conditions, related to the recent notion of robust Hadamard matrices, implies also the nonexistence of these circulant Hadamard matrices. If some of the conditions fail, it appears (to us) very difficult to be able to prove the result.
\end{abstract}

\section{INTRODUCTION}

A matrix of order $n$ is a square matrix with $n$ rows. A circulant matrix $A:=$ $\operatorname{circ}\left(a_{1}, \ldots, a_{n}\right)$ of order $n$ is a matrix of order $n$ of first row $\left[a_{1}, \ldots, a_{n}\right]$ in which each row after the first is obtained by a cyclic shift of its predecessor by one position. For example, the second row of $A$ is $\left[a_{n}, a_{1}, \ldots, a_{n-1}\right]$. A Hadamard matrix $H$ of order $n$ is a matrix of order $n$ with entries in $\{-1,1\}$ such that $K:=\frac{H}{\sqrt{n}}$ is an orthogonal matrix. A circulant Hadamard matrix of order $n$ is a circulant matrix that is Hadamard. The 10 known circulant Hadamard matrices are $H_{1}:=\operatorname{circ}(1), H_{2}:=$ $-H_{1}, H_{3}:=\operatorname{circ}(1,-1,-1,-1), H_{4}:=-H_{3}, H_{5}:=\operatorname{circ}(-1,1,-1,-1), H_{6}:=-H_{5}$, $H_{7}:=\operatorname{circ}(-1,-1,1,-1), H_{8}:=-H_{7}, H_{9}:=\operatorname{circ}(-1,-1,-1,1), H_{10}:=-H_{9}$.

If $H=\operatorname{circ}\left(h_{1}, \ldots, h_{n}\right)$ is a circulant Hadamard matrix of order $n$ then its representer polynomial is the polynomial $R(x):=h_{1}+h_{2} x+\cdots+h_{n} x^{n-1}$.

Key words and phrases. Circulant matrices, Hadamard matrices, eigenvalues, unit circle, cyclotomic fields.

2010 Mathematics Subject Classification. Primary: 11B30. Secondary: 15B34, 11R18.

DOI 10.46793/KgJMat2105.751E

Received: February 25, 2019.

Accepted: May 20, 2019. 
No one has been able, despite several deep computations (see [1,14]), to discover any other circulant Hadamard matrix. Ryser proposed in 1963 (see [16], [3, p. 97]) the conjecture of the non-existence of these matrices when $n>4$. Preceding work on the conjecture includes $[4,5,8-11,13,15,18]$. Ryser's conjecture above has been studied with several different methods. The first special case done by Brualdi [2] in 1965, assumed that all eigenvalues of $H:=\operatorname{circ}\left(h_{1}, \ldots, h_{n}\right)$, a circulant Hadamard matrix of order $n>4$, were real, i.e., that $H$ is symmetric, or equivalently that

$$
h_{n-k}-h_{k+2}=0, \quad \text { for } k=1, \ldots, \frac{n}{2}-2 .
$$

Besides Brualdi's result, all other known results are only partial results for particular $n$ 's, generally obtained by deep methods: see Turyn's work [18] and e.g., [15]. For example, the known case where $n$ has two prime factors, i.e., $n=4 p^{2 m}$ for some odd prime number $p$, is a consequence of some results of Turyn. These results permitted some computer calculations (e.g., in citations above) that proved the result for increasing numerical values of $n$. However, these methods seem to be unable to produce general proofs (say a proof of the conjecture for an infinity of $n$ 's with more than two prime factors).

The object of the present paper is to prove the conjecture in some new special cases related to some properties of eigenvalues of a possible new circulant Hadamard matrix, generalizing some properties of the 8 circulant Hadamard matrices of order 4 . Indeed, we prove that these properties hold only for $n=4$ assuming that they hold for $n \geq 4$. Essentially we prove that circulant Hadamard matrices of order $n>4$ cannot "inherit" some "linear" and "count" properties of the known circulant Hadamard matrices of order 4. To prove the full conjecture is equivalent to find a procedure that do not depends on conditions. Thus, we (and many other people in this area) are far from attaining this goal.

In practice, and more precisely, first, we prove (in Theorem 1.1 below) the result by replacing the equalities (1.1) on the $h_{i}$ 's by an upper bound on the number of similar equalities.

Theorem 1.1. Let $H=\operatorname{circ}\left(h_{1}, \ldots, h_{n}\right)$ be a circulant Hadamard matrix of order $n \geq 4$. Then $n=4$ provided the number $r$ of $i$ 's between 1 and $n / 2$ such that $h_{i}+h_{n / 2+i}=0$ does not exceed $\sqrt{n} / 2$.

Remark 1.1. When $n=4$ the condition of Theorem 1.1 holds, with $r=1$, for all 8 circulant Hadamard matrices $H_{3}, \ldots, H_{10}$.

In our second result we replace the condition of Theorem 1.1 by a property of some appropriate (complex) arithmetic mean related to the eigenvalues of $H$.

Theorem 1.2. Let $H=\operatorname{circ}\left(h_{1}, \ldots, h_{n}\right)$ be a circulant Hadamard matrix of order $n \geq 4$. Let $\omega:=\exp (2 \pi i / n)$. Then $n=4$ provided both statements (a) and (b) below hold. 
(a) There exists $k \in\{1, \ldots, n\}$ such that $k \notin\{n, n / 2\}$, and for $v:=\omega^{k}$ there exists an $n$-tuple $S:=\left(\epsilon_{1}, \ldots, \epsilon_{n}\right)$, depending on $k$, where $\epsilon_{j} \in\{-1,1\}$, such that

$$
a:=\frac{\sum_{j=1}^{n} \epsilon_{j} h_{j} v^{j-1}}{n} \in \mathbb{Z}[\omega]
$$

(b) The set $T:=\left\{1 \leq j \leq n: \epsilon_{j}=-1\right\}$ satisfies $r:=\operatorname{card}(\mathrm{T}) \leq \sqrt{n} / 2$.

Remark 1.2. When $n=4$ and $H=\operatorname{circ}\left(h_{1}, h_{2}, h_{3}, h_{4}\right)$, the conditions on Theorem 1.2 hold with:

$$
\omega:=i, \quad \text { so that } \omega^{2}=-1, \quad k=1, \quad S=\left(h_{1}, h_{2}, h_{3}, h_{4}\right),
$$

so that $r=1$ for all 8 circulant Hadamard matrices of order 4 , namely for $H_{3}, \ldots, H_{10}$.

Finally, in our third main result, we consider properties of the circulant Hadamard matrices (namely: $(-1)$ robust, say type 1 Hadamard matrices) related to the recent notion [6] of robust Hadamard matrices More precisely, 4 of the 8 known circulant Hadamard matrices of order 4 are indeed $(-1)$ robust Hadamard matrices while the other 4 (call them weak Hadamard, say type 2 Hadamard matrices) have a strong opposite property on their principal minors, (see definitions of robust Hadamard matrices and of both types of Hadamard matrices in section 2) and see more details in Remark 1.3 below. We show then in the following theorem that, under some mild conditions, these properties hold for $n=4$ and not for $n>4$. Observe also (see again Theorem 1.3) that there is no circulant Hadamard matrices that are robust. This is the reason why we defined the related notions discussed above. Given any $n \times n$ matrix $M=\left(M_{i, j}\right)$, with $n \geq 2$, we denote, in all the paper, by $m(1, k)$ the principal $2 \times 2$ minor of $M$, i.e., the determinant of the $2 \times 2$ submatrix $S$ of $M$ such that $S_{1,1}=M_{1,1}, S_{2,1}=M_{1, k}, S_{1,2}=M_{k, 1}$ and $S_{2,2}=M_{k, k}$. Moreover, in all the paper $H^{*}$ means the (complex) conjugate transpose of the matrix $H$, so that $H^{*}$ coincides with the transpose $H^{T}$ when $H$ has real coefficients.

Theorem 1.3. Let $H=\operatorname{circ}\left(h_{1}, \ldots, h_{n}\right)$ be a circulant Hadamard matrix of order $n \geq 4$. Then statement (a) holds, and one has $n=4$ provided any of statements (b) or (c) below hold. We can assume without loss of generality that $h_{1}=1$.

(a) The matrix $H$ cannot be robust.

(b) The matrix $H$ is $(-1)$ robust.

(c) The matrix $H$ is weak, $h_{1}+h_{n / 2+1}=0$, the number $n_{1}$ of 1 's in the entries $h_{1}, \ldots, h_{n / 2}$ of $H$, equals $\frac{n+\sqrt{n}+2}{4}$ and the number $n_{-1}$ of -1 's inside the same entries equals $\frac{n-\sqrt{n}-2}{4}$.

Remark 1.3. When $n=4$ the four $(-1)$ robust Hadamard circulants are $H_{5}, H_{6}, H_{9}$ and $H_{10}$. Thus the 4 weak circulant Hadamard are $H_{3}, H_{4}, H_{7}$ and $H_{8}$.

Remark 1.4. For a general regular Hadamard matrix $H=\operatorname{circ}\left(h_{1}, \ldots, h_{n}\right)$, say with $h_{1}=1$, it is known (see Lemma 2.1) that the number of 1's in any row equals $r_{1}:=\frac{n+\sqrt{n}}{2}$. Since we consider type 2 matrices in part (c) of our last theorem it is 
natural to think, (but it is not proved, and might be difficult to prove), and has been nevertheless used as an hypothesis, that we should have about $r_{1} / 2$ entries equal to 1 in the first $\frac{n}{2}$ entries of the first row of $H$. The condition on Theorem 1.3, part (c) comes from this consideration, since it matches exactly the case of the 4 circulant matrix $H_{8}:=\operatorname{circ}(1,1,-1,1)$ where we have two 1 's and so zero -1 in the first two entries of the first row. The other 3 circulant Hadamard matrices of order 4 and type 2 , are obtained by shifts of length 2 of the first row of $H_{8}$, (see details, as before, in Remark 1.3).

The necessary tools for the proof of all three theorems are given in Section 2. The proof of Theorem 1.1 is presented in Section 3, the proof of Theorem 1.2 is presented in Section 4, and the proof of Theorem 1.3 is presented in Section 5.

\section{TOOLS}

The following is well known. See, e.g., [7, p. 1193], [12, p. 234], [18, p. 329-330].

Lemma 2.1. Let $H$ be a regular Hadamard matrix of order $n \geq 4$, i.e., a Hadamard matrix whose row and column sums are all equal. Then $n=4 h^{2}$ for some positive integer $h$. Moreover, the row and column sums are all equal to $\pm 2 h$ and each row has $2 h^{2} \pm h$ positive entries and $2 h^{2} \mp h$ negative entries. Finally, if $H$ is circulant then $h$ is odd.

Lemma 2.2. Let $H$ be a circulant Hadamard matrix of order $n$, let $w=\exp (2 \pi i / n)$ and let $R(x)$ be its representer polynomial. Then all the eigenvalues $R(v)$ of $H$, where $v \in\left\{1, w, w^{2}, \ldots, w^{n-1}\right\}$, satisfy $|R(v)|=\sqrt{n}$.

We recall here the definition of robust Hadamard matrices from [6] and define the notions of $(-1)$ robust and of weak Hadamard matrix.

Definition 2.1. Let $H$ be an Hadamard matrix of order $n$.

(a) We say that $H$ is robust if all $2 \times 2$ principal minors of $H$ are in $\{-2,2\}$.

(b) We say that $H$ is $(-1)$ robust if all $2 \times 2$ principal minors, but the minor $m(1, n-1)$ of $H$, that equals 0 , are in $\{-2,2\}$.

(c) We say that $H$ is weak if all $2 \times 2$ principal minors of $H$ equal 0 .

Remark 2.1. An Hadamard matrix $H$ is robust if and only if every principal $2 \times 2$ submatrix of $H$ is also an Hadamard matrix. An Hadamard matrix $H$ is weak if and only if every principal $2 \times 2$ submatrix of $H$ is singular. In order that a circulant Hadamard $H:=\operatorname{circ}\left(h_{1}, \ldots, h_{n}\right)$ matrix be robust (resp. weak) it is necessary and sufficient that the principal $2 \times 2$ submatrices with first column $\left[h_{1}, h_{k}\right]^{T}$ (where the $T$ means "transpose") be Hadamard (resp. be singular).

The next lemma (see [17, Lemma 8.6]) is frequently used in the theory of group representations. Here, it is useful for the proof of Lemma 2.4. 
Lemma 2.3. Let $c_{1}, \ldots, c_{\ell}$ be $\ell$ complex numbers of absolute value 1 . If $\left|c_{1}+\cdots+c_{\ell}\right|=\ell$, then $c_{1}=\cdots=c_{\ell}$.

The next lemma is about some complex arithmetic means.

Lemma 2.4. Let $\omega:=\exp (2 \pi i / n)$. Let $c_{1}, \ldots, c_{n}$ be $n$ elements of $\mathbb{Z}[\omega]$ of absolute value 1 . If

$$
\frac{c_{1}+\cdots+c_{n}}{n} \in \mathbb{Z}[\omega],
$$

then either $c_{1}=\cdots=c_{n}$ or $c_{1}+\cdots+c_{n}=0$.

Proof. Put $a:=\frac{c_{1}+\cdots+c_{n}}{n}$. The hypothesis implies that $|a| \leq 1$. If at least two of the $c_{j}$ 's are distinct, then by Lemma 2.3 (with $\ell=n$ ) we get $|a|<1$ so that $|\sigma(a)|<1$ for any $\sigma \in G$, where $G:=\operatorname{Gal}(\mathbb{Q}(\omega) / \mathbb{Q})$ is the Galois group of the cyclotomic field $\mathbb{Q}(\omega)$ over $\mathbb{Q}$. Thus $P:=\prod_{\sigma \in G} \sigma(a) \in \mathbb{Z}$ satisfies $0 \leq|P|<1$. It follows that $P=0$, so that $a=0$.

\section{Proof of Theorem 1.1}

Put $w:=\exp (2 \pi i / n)$. Observe that $H$ is regular in terms of Lemma 2.1 since $H$ is circulant. In particular, Lemma 2.1 implies that $n=4 h^{2}$ for some positive integer $h$. Write $H=\operatorname{circ}\left(h_{1}, \ldots, h_{n}\right)$ and let $R(x)$ be the representer polynomial of $H$. By Lemma 2.2 one has $R(w)=2 h a$ where $a$ is a complex number in the unit circle. Let $W:=\left\{j=1, \ldots \frac{n}{2}: h_{j}=-h_{n / 2+j}\right\}$ and let

$$
t:=\sum_{j \in W} h_{j} \omega^{j-1}
$$

Then one has

$$
2 h a-2 t=z_{1}+\cdots+z_{n},
$$

where

$$
z_{j}:=h_{j} \omega^{j-1}, \quad \text { for all } j=1, \ldots, \frac{n}{2} \text { such that } j \notin W
$$

and

$$
z_{j}:=-h_{j} \omega^{j-1}, \quad \text { for all } j=1, \ldots, \frac{n}{2} \text { such that } j \in W,
$$

and

$$
z_{n / 2+j}:=h_{n / 2+j} \omega^{n / 2+j-1}, \quad \text { for all } j=1, \ldots, \frac{n}{2} .
$$

Since $\omega^{n / 2}=-1$, we see that (3.3), (3.4) and (3.5) guarantee that

$$
z_{n / 2+j}=-z_{j}, \quad \text { for all } j=1, \ldots, \frac{n}{2} .
$$

More precisely, if $j \notin W$ then $z_{j}=h_{j} \omega^{j-1}$, while $z_{n / 2+j}=h_{n / 2+j} \omega^{n / 2+j-1}=$ $h_{j} \omega^{n / 2} \omega^{j-1}=-h_{j} \omega^{j-1}=-z_{j}$. If $j \in W$ then $z_{j}=-h_{j} \omega^{j-1}$, while $z_{n / 2+j}=$ $h_{n / 2+j} \omega^{n / 2+j-1}=-h_{j} \omega^{n / 2} \omega^{j-1}=h_{j} \omega^{j-1}=-z_{j}$. 
Since $\omega \notin \mathbb{R}$ and $\omega$ has multiplicative order equal to $n$ it follows from (3.6) that we have $z_{i} \neq z_{j}$ for all $i \neq j, 1 \leq i, j \leq n$.

It then follows from (3.6) that

$$
z_{1}+z_{2}+\cdots+z_{n}=0
$$

But by (3.2), we see that (3.7) implies

$$
\sqrt{n} a=2 t .
$$

But $|a|=1$, and by hypothesis $\operatorname{card}(\mathrm{W}) \leq \sqrt{n} / 2$, thus it follows from (3.8) and from the definition of $t$ in (3.1) that

$$
\frac{\sqrt{n}}{2}=|t| \leq \operatorname{card}(\mathrm{W}) \leq \frac{\sqrt{n}}{2}
$$

so that

$$
\frac{\sqrt{n}}{2}=|t|=\left|\sum_{j \in W} h_{j} \omega^{j-1}\right|=\operatorname{card}(\mathrm{W}) .
$$

Put for every $j \in W, d_{j}:=h_{j} \omega^{j-1}$. Since $\left|d_{j}\right|=1$ for all these $j$ 's, it follows from (3.9) and from Lemma 2.3 (with $\ell=\sqrt{n} / 2$ ) that

$$
d_{i}=d_{j}, \quad \text { for all } i, j \in W .
$$

Assume now that $n>4$. Then (3.10) is impossible since $\omega^{i-1} \neq \pm \omega^{j-1}$ when $i \neq j$ for any $i, j \in\left\{1,2, \ldots, \frac{n}{2}\right\}$. Therefore, $n=4$. This finishes the proof of Theorem 1.1.

\section{Proof of Theorem 1.2}

We refer to notations in Theorem 1.2. From Lemma 2.2, $\lambda$ defined by

$$
\lambda:=h_{1}+h_{2} v+\cdots+h_{n} v^{n-1},
$$

where $v=\omega^{k}$, is an eigenvalue of $H$. By the same Lemma 2.2, $\lambda$ satisfies $|\lambda|=\sqrt{n}$.

Observe that $T$ is not empty, since $T=\varnothing$ implies $a=\lambda / n$ so that $|a|=1 / \sqrt{n}$ since by Lemma $2.2|\lambda|=\sqrt{n}$. But hypothesis (a) implies that the complex conjugate $\bar{a} \in \mathbb{Z}[\omega]$ so that $1 / n=|a|^{2}=a \bar{a} \in \mathbb{Z}[\omega]$. Therefore, we get the contradiction that $n=1$. One has by hypothesis (a) and by (4.1)

$$
\lambda-n a=2 \sum_{i \in T} h_{i} v^{i-1} .
$$

Putting $c_{j}=\epsilon_{j} h_{j} v^{j-1}$ for all $j=1 \ldots n$, it is clear that $n a=c_{1}+\cdots+c_{n}, c_{j} \in \mathbb{Z}[\omega]$, and that $\left|c_{j}\right|=1$ for all these $j$ 's. Moreover, $k \notin\{n, n / 2\}$ implies that $v \notin \mathbb{R}$ so that $c_{1} \neq c_{2}$.

It follows then from Lemma 2.4 that $a=0$. Thus, from (4.2) we get

$$
\lambda=2 s, \quad \text { where } s=\sum_{i \in T} h_{i} v^{i-1} .
$$


Now, Lemma 2.2 and (4.3) imply that

$$
|s|=\frac{\sqrt{n}}{2} .
$$

But from the definition of $s$ in (4.3) and the triangular inequality one has

$$
|s| \leq \sum_{i \in T}\left|h_{i} v^{i-1}\right|=\sum_{i \in T} 1=\operatorname{card}(\mathrm{T}) .
$$

From (4.5), (4.4) and hypothesis (b) we obtain

$$
|s|=\operatorname{card}(\mathrm{T})=\frac{\sqrt{n}}{2} .
$$

Putting $d_{j}:=h_{j} v^{j-1}$ for all $j \in T$, it is clear that $\left|d_{j}\right|=1$ for all these $\frac{\sqrt{n}}{2}$ values of $j$. Thus from (4.6) and from Lemma 2.3 (with $\ell=\sqrt{n} / 2$ ) we obtain that

$$
d_{i}=d_{j}, \quad \text { for all } j \in T \text {. }
$$

Remember that, by Lemma 2.1, $n=4 h^{2}$ with odd $h$. By (4.6), $h=$ card(T). Thus, if $\operatorname{card}(\mathrm{T})>1$ then $h=\operatorname{card}(\mathrm{T}) \geq 3$ so that $(4.7)$ cannot hold since $\omega^{n / 2}=-1$ implies that for $i, j \in T$, with $i \leq j$

$$
d_{i}=d_{j} \Longleftrightarrow i=j \text { or } j=i+\frac{n}{2} \text {. }
$$

In other words, (4.8) says that there cannot exist three elements $i, j, k \in T$ that are 2 by 2 distinct and for which $d_{i}=d_{j}=d_{k}$. Thus, $\operatorname{card}(\mathrm{T})=1$, that is, from (4.6), we have $n=4$. This proves the theorem.

\section{Proof of Theorem 1.3}

Part (a). Assume, to the contrary, that $H$ is robust. It follows from the following equality (see [6, Formula (3.5) in proof of Lemma 3.6, Subsection 3.3]) that:

$$
H D^{*}+D H^{*}=2 I,
$$

where $D$ is the diagonal matrix containing the diagonal elements of $H$, i.e., in our case $D=I$ so that (5.1) becomes

$$
H+H^{*}=2 I .
$$

But, multiplying both sides of (5.2) by the eigenvector $v:=R(1)=[1,1, \ldots, 1]^{*}$ of $H$, (see Lemma 2.2) we get $2 \sqrt{n} v=2 v$, i.e., we get the contradiction $n=1$.

The following observation is useful for the proof of parts (b) and (c): $H:=\left(h_{i, j}\right)=$ $\operatorname{circ}\left(h_{1}, \ldots, h_{n}\right)$ if and only if the following condition on the indices $(\bmod n)$ holds

$$
h_{i, j}=h_{j-i+1}(\bmod n) \cdot
$$

Part (b). Assume to the contrary, that $n>4$. Observe that by Lemma 2.1 we can assume that

$$
n \geq 36 \text {. }
$$


Since $H$ is $(-1)$ robust one has $m(1, j)=2$ for all $j=2, \ldots, n-2, m(1, n-1)=0$ and $m(1, n)=2$. In other words, (and by using (5.3)) we have $h_{j} h_{n-j+2}=-1$ for all $j=2, \ldots, n-2, h_{3} h_{n-1}=1$ and $h_{2} h_{n}=-1$. This can also be written as: $h_{n-j+2}=-h j$ for all $j=2, \ldots, n-2, h_{n-1}=h_{3}$ and $h_{n}=-h_{2}$. Thus we can write the relation $\sqrt{n}=R(1)$ as follows

$$
\sqrt{n}=h_{1}+\sum_{j=2, j \neq 3}^{n / 2+1} h_{j}-\left(\sum_{t=2, t \neq 3}^{n / 2} h_{t}\right)+h_{3}+h_{3} .
$$

Writing (5.5) in the following form

$$
\sqrt{n}=h_{1}+h_{n / 2+1}+\sum_{j=2, j \neq 3}^{n / 2} h_{j}-\left(\sum_{t=2, t \neq 3}^{n / 2} h_{t}\right)+h_{3}+h_{3},
$$

it is clear that we get

$$
\sqrt{n}=h_{1}+h_{n / 2+1}+2 h_{3}
$$

so that

$$
\sqrt{n}=\left|h_{1}+h_{n / 2+1}+2 h_{3}\right| \leq 4 .
$$

But, (5.6) contradicts (5.4), thereby finishing the proof of part (b).

Part (c). Assume, to the contrary, that $n>4$. Let $s:=\sum_{k=2}^{n / 2} h_{k}$. Proceeding as before we get now

$$
\sqrt{n}=R(1)=h_{1}+h_{n / 2+1}+2 s,
$$

since now we have $h_{n-j-2}=h_{j}$ for all $j=2, \ldots, n$. Let us compute now $s$ by using our hypothesis on the number of 1's and -1 's in the $h_{j}$ 's, with $j=1, \ldots \frac{n}{2}$,

$$
s=m_{1}-m_{-1}=\frac{2 \sqrt{n}+4}{4}
$$

thus (5.7) becomes

$$
\sqrt{n}=h_{1}+h_{n / 2+1}+\sqrt{n}+2 .
$$

We have then from (5.8)

$$
h_{1}=h_{n / 2+1}=-1 .
$$

But, (5.9) contradicts our hypothesis $h_{1}+h_{n / 2+1}=0$, thereby proving part (c). This proves the theorem.

\section{REFERENCES}

[1] P. Borwein and M. J. Mossinghoff, Wieferich pairs and Barker sequences II, LMS J. Comput. Math. 17(1) (2014), 24-32.

[2] R. A. Brualdi, A note on multipliers of difference sets, Journal of Research of the National Bureau of Standards, Section B 69 (1965), 87-89.

[3] P. J. Davis, Circulant Matrices, 2nd ed., AMS Chelsea Publishing, New York, 1994.

[4] R. Euler, L. H. Gallardo and O. Rahavandrainy, Sufficient conditions for a conjecture of Ryser about Hadamard Circulant matrices, Linear Algebra Appl. 437 (2012), 2877-2886. 
[5] R. Euler, L. H. Gallardo and O. Rahavandrainy, Combinatorial properties of circulant Hadamard matrices, in: C. M. da Fonseca, D. Van Huynh, S. Kirkland and V. K. Tuan (Eds.), A panorama of Mathematics: Pure and Applied, Contemporary Mathematics (Book 658), American Mathematical Society, Providence, RI, 2016, 9-19.

[6] A. Gąsiorowski, G. Rajchel and K. Zyczkowski, Robust Hadamard matrices, unistochastic rays in Birkhoff polytope and equi-entangled bases in composite spaces, Math. Comput. Sci. 12(4) (2018), 473-490.

[7] A. Hedayat and W. D. Wallis, Hadamard matrices and their applications, Ann. Statist. 6(6) (1978), 1184-1238.

[8] J. Jedwab and S. Lloyd, A note on the nonexistence of Barker sequences, Des. Codes Cryptogr. 2(1) (1992), 93-97.

[9] L. Gallardo, On a special case of a conjecture of Ryser about Hadamard circulant matrices, Appl. Math. E-Notes 12 (2012), 182-188.

[10] L. H. Gallardo, New duality operator for complex circulant matrices and a conjecture of Ryser, Electron. J. Combin. 23(1) (2016), Paper ID 1.59, 10 pages.

[11] M. Matolcsi, A Walsh-Fourier approach to the circulant Hadamard conjecture, in: Algebraic Design Theory and Hadamard Matrices, Springer Proc. Math. Stat. 133, Springer, Cham, 2015, 201-208.

[12] D. B. Meisner, On a construction of regular Hadamard matrices, Atti della Accademia Nazionale dei Lincei. Classe di Scienze Fisiche, Matematiche e Naturali. Rendiconti Lincei. Serie IX. Matematica e Applicazioni 3(4) (1992), 233-240.

[13] Y. Y. Ng, Cyclic Menon difference sets, circulant hadamard matrices and Barker sequences, Master Thesis, The University of Hong Kong, December 1993, 36 pages.

[14] M. J. Mossinghoff, Wieferich prime pairs, Barker sequences, and circulant Hadamard matrices, 2013, [http://www.cecm.sfu.ca/ mjm/WieferichBarker/].

[15] K. H. Leung and B. Schmidt, New restrictions on possible orders of circulant Hadamard matrices, Des. Codes Cryptogr. 64 (2012), 143-151.

[16] H. J. Ryser, Combinatorial Mathematics, The Carus Mathematical Monographs 14, The Mathematical Association of America, John Wiley and Sons, Inc., New York, 1963.

[17] J.-P. Serre, Finite Groups: An Introduction, Surveys of Modern Mathematics 10, International Press, Somerville, MA, Higher Education Press, Beijing, 2016.

[18] R. J. Turyn, Character sums and difference sets, Pac. J. Math. 15 (1965), 319-346.

${ }^{1}$ LAB-STICC CNRS UMR 6285, COMPUTER SCIENCE,

UNIVERSITY OF BREST,

20, Av. Le Gorgeu, C.S. 93837, 29238 Brest Cedex 3, France

Email address: Reinhardt.Euler@univ-brest.fr

${ }^{2}$ Department of Mathematics,

UNIVERSITY OF BREST,

6, Av. Le Gorgeu, C.S. 93837, 29238 Brest Cedex 3, France

Email address: Luis.Gallardo@univ-brest.fr

Email address: Olivier.Rahavandrainy@univ-brest.fr 